\title{
Study on automatic detection method for the enclosed turbine runner chamber based on real-time image acquisition and simulation comparison techniques
}

\author{
Yunlong Liu ${ }^{a}$, Xiangwei Yang, Dengke Li, Dingjie Shen and Jianwei Zou \\ State Grid Hunan Electric Power Company Research Institute, Changsha 410007, China
}

\begin{abstract}
According to the technical problems of the existing testing methods for the key hidden metal components (such as the decompression panel and the water board) of the turbine runner chamber, such as the poor reliability, the inaccurate locating and the larger detection blind spots of the detection device, under the downtime without opening the cover of the hydropower turbine runner chamber, an automatic detection method for the enclosed turbine runner chamber based on real-time image acquisition and simulation comparison techniques was proposed. The magnetic crawler with permanent magnet wheel could carry the real-time image acquisition device to complete the crawling work on the inner surface of the enclosed chamber, and collected the real-time scene image of the enclosed chamber. According to the obtained location by using the positioning auxiliary device, the position of the real-time detection image in a virtual 3-D model was calibrated. Through comparing of the real-time detection images and the computer simulation images, the defects or foreign matter fall into could be accurately positioning, so as to repair and clean up conveniently. It has important practical value to realize the automatic detection of the difficult demolition and complicated metal components of the enclosed turbine runner chamber, to ensure the safety and reliability of operation.
\end{abstract}

Keywords: real-time image acquisition; simulation comparison; decompression panel; water board; automatic detection; enclosed turbine runner chamber.

\section{Introduction}

Turbine rubbing fault (such as decompression panel drop) often lead to the abnormal vibration, damaged structures, and even can cause splitting cover and flooding plant accident. A plurality of hydropower plants at home and abroad have been occurred serious accidents, such as cover rubbing and damaged fault [1]. For example, more than 74 lives deprived by the flooding plant serious accident because of the cover damage at the Russia Sayano-Shushenskaya hydroelectric power station. Besides, the splitting cover and flooding plant accident has been frequent occurred at Ankang, Yantan, Danjiangkou and Panjiakou etc. Hydropower plants [2].

To prevent major accidents of the turbine, it is necessary to carry out the technology research of the fault detection and protection monitoring for the key components under the turbine cover. However, it is a time-consuming and labor-intensive work to open the cover of the turbine runner chamber [3]. So the

\footnotetext{
${ }^{\mathrm{a}}$ Corresponding author : 13874809964@163.com
} 
cover is just opened during A-type repair. When making B-type or C-type repair in hydropower plant, the endoscope is often used to extended into the chamber under the closed cover from the vacuum breaking valve hole above or the relief hole below to observe the key components such as the decompression panel and the water board in the enclosed turbine runner chamber. Since the endoscope hose manual operation, the length and angle of the inserted probe cannot be controlled, it is difficult to determine the specific location of the inner chamber of the detection probe. Therefore, it is impossible to determine the position and properties accurately. Even if the defects or foreign matter is observed, it also cannot effectively guide the maintenance staff to repair. In addition, there is a big blind spot observation [4].

In this paper, an automatic detection method for the enclosed turbine runner chamber based on real-time image acquisition and simulation comparison techniques was proposed. The magnetic crawler with permanent magnet wheel could carry the real-time image acquisition device to complete the crawling work on the inner surface of the enclosed chamber. Then the image acquisition device completed the real-time collection of the scene image of the enclosed chamber. According to the obtained location by using the positioning auxiliary device, the position of the real-time detection image in a virtual 3-D model was calibrated. Through comparing of the real-time detection images and the computer simulation images, the defects or foreign matter fall into could be accurately positioning, so as to repair and clean up conveniently. It has important practical value to realize the automatic detection of the difficult demolition and complicated metal components of the enclosed turbine runner chamber, to solve the detection problems of common concern of hydropower manufacturing industry at home and abroad.

\section{Principle of automatic detection system}

The components of the enclosed turbine runner chamber automatic detection system are shown in Fig. 1, which contains the computer simulation comparison and control systems, the integrated module for data acquisition and processing, the magnetic crawler, the steering and transmission device, the positioning and distance measuring device, the image acquisition and illumination device and the DC power supply.
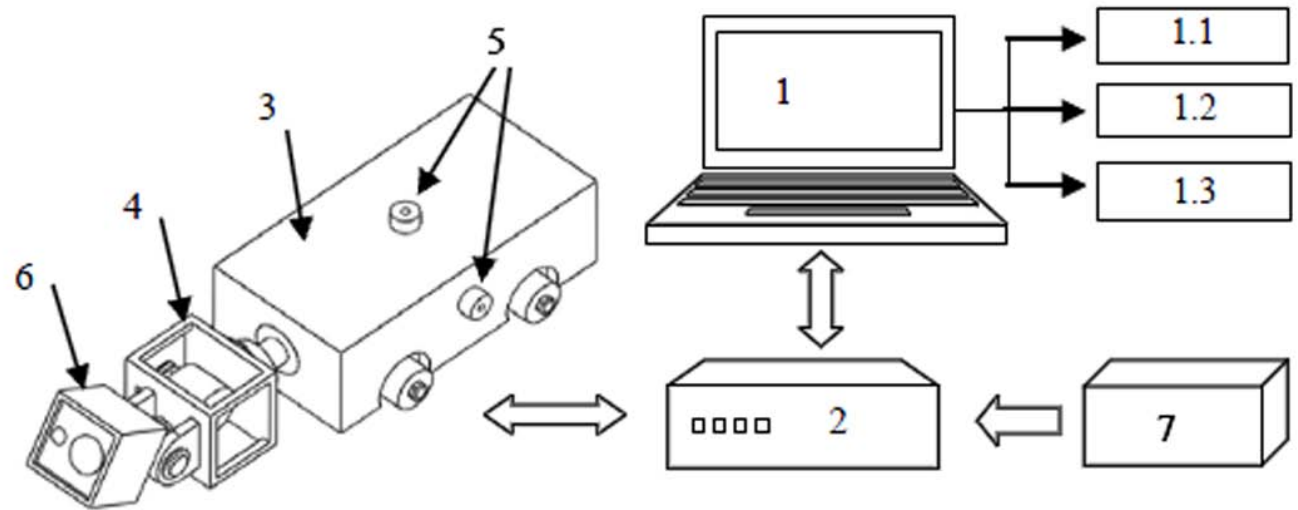

1- computer control systems (1.1- motor control system, 1.2- simulation comparison system, 1.3- data acquisition and processing system), 2- integrated module for data acquisition and processing, 3- magnetic crawler, 4- steering and transmission device, 5- positioning and distance measuring device, 6- image acquisition and illumination device, 7- DC power supply.

Figure 1. Components of the automatic detection system

The magnetic crawler with permanent magnet wheel could carry the real-time image acquisition device to complete the crawling work on the inner surface of the enclosed chamber. Then the image acquisition device is used to complete the real-time collection of the scene image of the enclosed chamber, and the positioning and distance measuring device is used to achieve the positioning of the 
crawler. According to the obtained location by using the positioning auxiliary device, the position of the real-time detection image in a virtual 3-D model was calibrated. Through comparing of the real-time detection images and the computer simulation images, the defects or foreign matter fall into could be accurately positioning, in order to clean up and eliminate system error conveniently.

\section{Design of the control system}

The flow chat of the detection control system is shown in Fig. 2, which contains 4 motors, and half of them are used to control the motion of the magnetic crawler, and the others are used to achieve the perspective control of the image acquisition and illumination device. The magnetic crawler has 2 driving wheels (or rear wheels) and 2 driven wheels (or front wheels). The driving wheel and the driven wheel on the same side are connected with the belt and the 2 driving wheels are controlled by 2 motors respectively. Through coordinated control of the 2 motors, it could be realized to control the magnetic crawler to forward, backward and steering.The simulation comparison system is used to establish the 3-D structural model of the enclosed turbine runner chamber. By using the positioning and distance measuring device, the characteristic parameters which contain the real-time position coordinates of the magnetic crawler and the acquired image could be obtained. Then the observation point and the position of the image could be calibrated in the corresponding 3-D simulation model, to realize the split-screen display of the real-time acquired image and the simulation image.

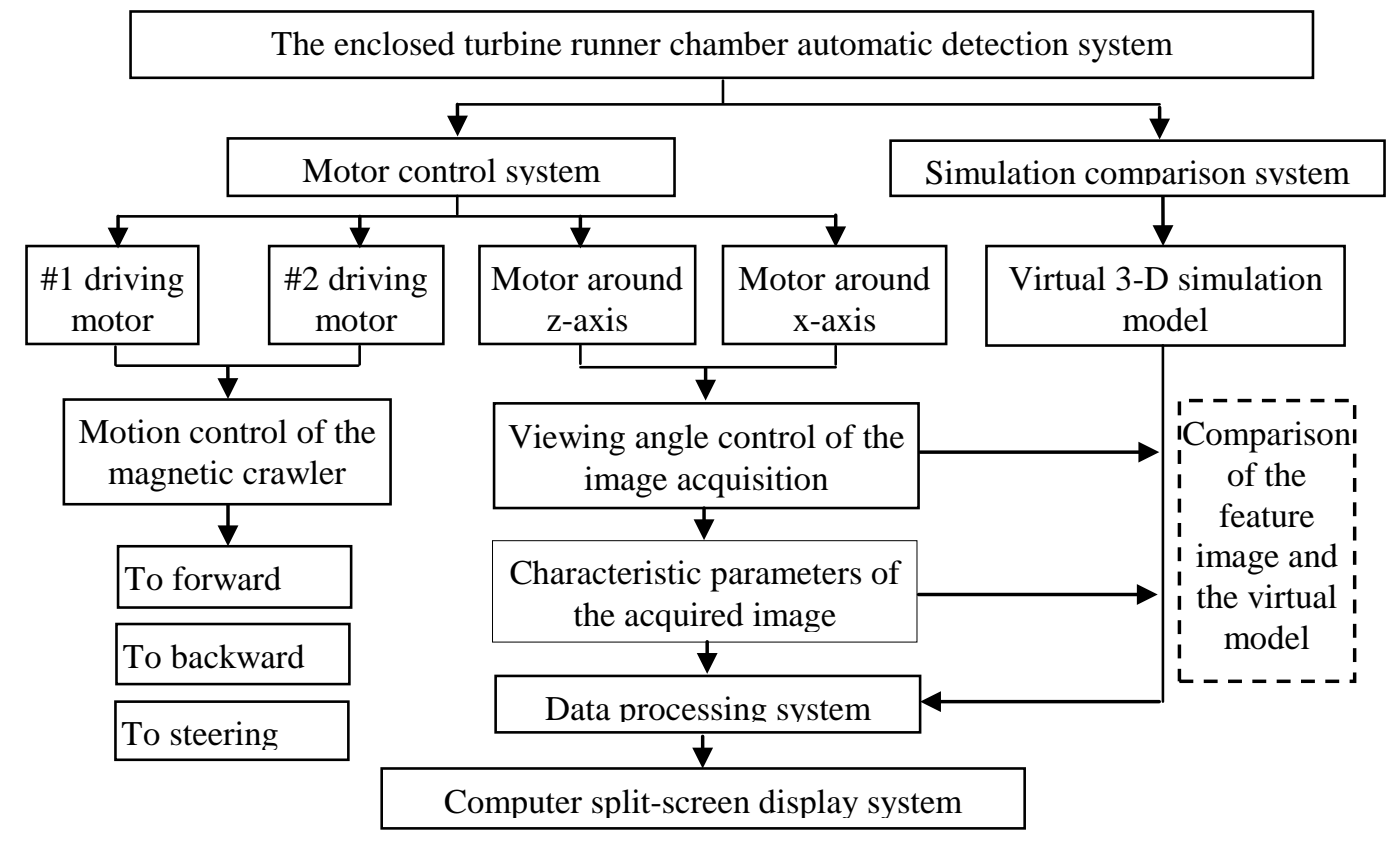

Figure 2 . The flow chat of the detection control system

\section{Split-screen display control}

According to the positioning and distance measuring device, the real-time acquisition feature image could be positioned. Then the position of the corresponding feature image in the virtual model could be calibrated and the real-time acquisition feature image and the corresponding feature image in the virtual model could be split-screen display in the computer control interface. As a result, the corresponding virtual image with the same size virtual foreign matter would be displayed on another screen at the same time. 


\section{Application test analysis}

Taking the enclosed square vessel as an example, the working process of the automatic detection system was analyzed, as shown in Fig 3 and Fig 4.

Firstly, the magnetic crawler which carries the real-time image acquisition device and the positioning measuring device was put into the test enclosed square vessel, as shown in Fig 3. According to the collected positional parameters (A, B and $\mathrm{C}$ ) by using the positioning measuring device, the magnetic crawler could be positioned; according to the acquired angle parameters ( $\alpha$ and $\beta$ ) between the machine vision centerline and $\mathrm{x}$-axis and $\mathrm{y}$-axis, the real-time acquisition feature image could be positioned.

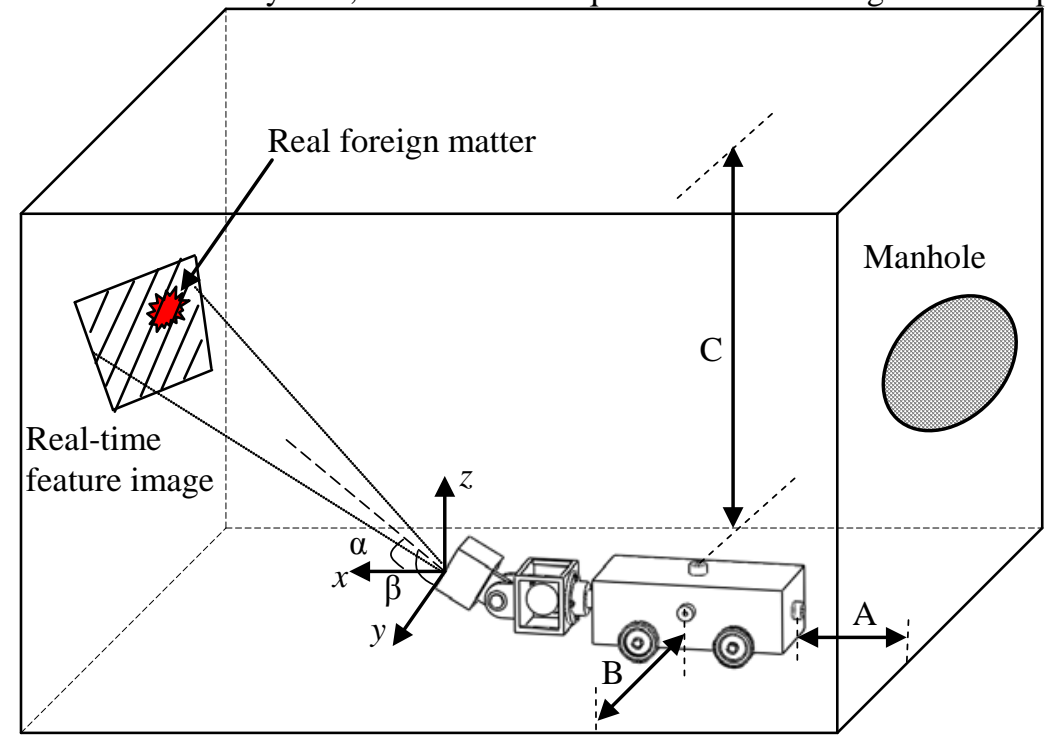

Figure 3. The field application diagram of the enclosed vessel

Secondly, the virtual 3-D model of the test enclosed square vessel was established and the position of the corresponding feature image in the virtual model could be calibrated, according to the obtained positional parameters (A, B and C), the angle parameters ( $\alpha$ and $\beta$ ) and the structure parameters (a, b, c, $\mathrm{n}$ and $\mathrm{m}$ ), as shown in Fig 4 . Then, the real-time acquisition feature image and the corresponding feature image in the virtual model could be split-screen display in the computer control interface. At this time, once a foreign matter was detected by the image acquisition device, it would be displayed on the real-time image display screen. Through comparing of the real-time images and the virtual model, the corresponding virtual image with the same size virtual foreign matter would be displayed on another screen at the same time.

Finally, the position and size of the defects or foreign matters fall into the enclosed square vessel could be obtained and be accurately calibrated, so as to repair and clean up conveniently. 


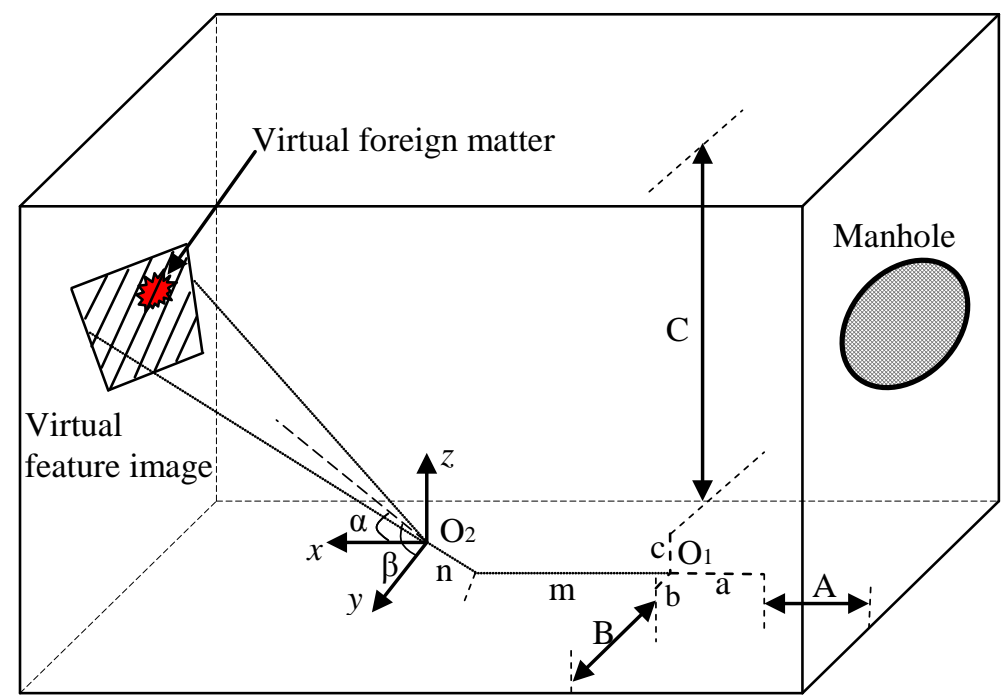

Figure 4. The schematic of image positioning of the virtual 3-D model

\section{Summary}

By proposing the automatic detection method, the problems of the existing testing methods for the key hidden metal components was improved significantly. The application test analysis showed that the proposed automatic detection device could calibrate accurately and clean up the defects or foreign matter conveniently without opening the cover of the enclosed hydropower turbine runner chamber. According to the existing testing technical problems for the key hidden metal components of the turbine runner chamber, such as the poor reliability and the larger detection blind spots, it is proved to be effective of the proposed automatic detection method by using the simulation comparison system.

\section{Acknowledgement}

This research was financially supported by the State Grid Hunan Electric Power Company (NO. 5216A514004A).

\section{References}

1. LIAO Tingting, CHEN Hechun and WANG Jibao. Analysis of Francis Turbine Blade Abrasion Principle for Three Gorges Hydropower Plant, International Journal Hydroelectric Energy. 29(2011) 118-120.

2. TANG Hua. Rebirth of Russia Sayano-Shushenskaya hydroelectric power station, Energy. 12(2014)20-23.

3. HE Cheng-bing, JIANG Di, ZHANG Ya-fei, et al. Analysis and Process on Rub-impact Fault of Large Turbine Generator Units, Turbine Technology. 1(2013) 47-50.

4. ZHANG Liang. Study on troubleshooting approach of turbine during running, China Hi-tech Enterprises. 0(2015)61-62. 